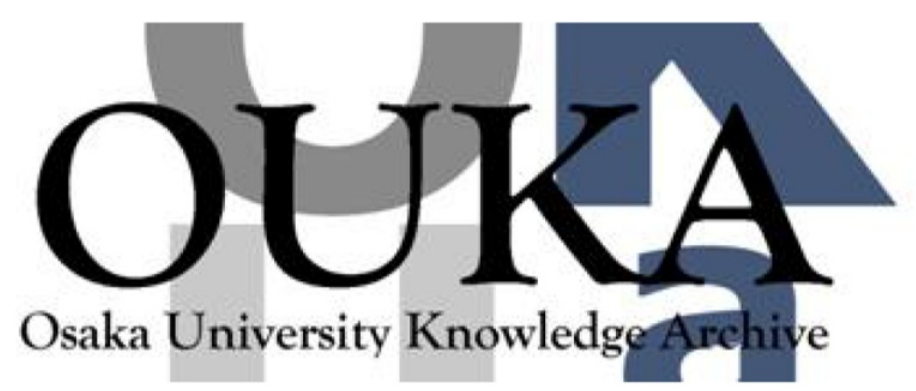

\begin{tabular}{|c|c|}
\hline Title & $\begin{array}{l}\text { Adaptive visualization of gas distribution } \\
\text { using augmented reality glasses }\end{array}$ \\
\hline Author(s) & $\begin{array}{l}\text { Duan, Liyuan; Matsukura, Haruka; Punpongsanon, } \\
\text { Parinya et al. }\end{array}$ \\
\hline Citation & $\begin{array}{l}2020 \text { IEEE 9th Global Conference on Consumer } \\
\text { Electronics, GCCE 2020. p. } 658-\text { p. } 659\end{array}$ \\
\hline Issue Date & 2020 \\
\hline oaire:version & AM \\
\hline URL & https://hdl. handle. net/11094/81843 \\
\hline rights & $\begin{array}{l}\text { ( } 2020 \text { IEEE. Personal use of this material is } \\
\text { permitted. Permission from IEEE must be } \\
\text { obtained for all other uses, in any current or } \\
\text { future media, including reprinting/republishing } \\
\text { this material for advertising or promotional } \\
\text { purposes, creating new collective works, for } \\
\text { resale or redistribution to servers or lists, } \\
\text { or reuse of any copyrighted component of this } \\
\text { work in other works. }\end{array}$ \\
\hline Note & \\
\hline
\end{tabular}

Osaka University Knowledge Archive : OUKA

https://ir. Library. osaka-u. ac. jp/

Osaka University 


\title{
Adaptive Visualization of Gas Distribution Using Augmented Reality Glasses
}

\author{
Liyuan Duan, Haruka Matsukura, Parinya Punpongsanon, Takefumi Hiraki, Daisuke Iwai, and Kosuke Sato \\ Graduate School of Engineering Science, Osaka University, Toyonaka, Osaka, Japan \\ \{liyuan, hiraki\}@sens.sys.es.osaka-u.ac.jp, \{haruka.matsu, parinya, daisuke.iwai, sato\}@ sys.es.osaka-u.ac.jp
}

\begin{abstract}
Monitoring gas distribution is a method to supervise and validate the stabilization and safety of landfill sites. Recent technologies that use sophisticated sensors confuse workers' understanding of estimated gas distribution due to mismatch between the gas position in a scene and the one in a monitor. In this paper, we propose a method for spatially visualizing gas distribution using augmented reality glasses. Furthermore, we discuss the initial step toward the adaptive visualization of gas distribution based on workers' knowledge and monitoring purposes using real-time AR.

Index Terms-Gas distribution mapping, Gas visualization, Augmented reality, Head-Mounted display
\end{abstract}

\section{INTRODUCTION}

Monitoring gas distribution is a way to supervise and validate the stabilization and safety of landfill sites. Recent advances in the visualization of gas distribution are being developed through the integration of monitors, cameras, GPS devices, and specific sensors. For example, an optical-based gas sensor can be used to measure methane gas, and this can be reconstructed and displayed in three-dimensional (3D) visualization [1]. Workers are required to observe detected gas visualized on monitors; however, this can confuse workers about the actual location of the gas in a real scene.

Augmented Reality (AR) can be used to convert gas distribution from specific sensors into a visualized form augmented in a real environment [2]. Although previous studies have been conducted in this regard, including the visualization of underground infrastructure [3], [4] and urban planning [5], studies on appropriate visualization techniques for workers in specific scenarios, such as landfill sites, have not been conducted. In this paper, we propose a model for exploring appropriate visualization techniques to represent gas distributions based on workers' knowledge and monitoring purposes. This work is an initial step toward an adaptive visualization of gas distribution using AR glasses.

\section{Adaptive Gas Visualization}

\section{A. Measuring Device and Display Device}

In our initial study, we apply our method with methane gas as similar as our previous study [1]. We used a gas detector to detect the gas distribution, and an AR Head-Mounted Display (AR-HMD) visualizes the gas in the near real-time (Fig. 1). A user wears an AR-HMD (Microsoft HoloLens) and holds a methane detector (Laser Methane mini SA3C32B-BJ, Tokyo Gas Engineering Solution), hereinafter referred to as LMm.

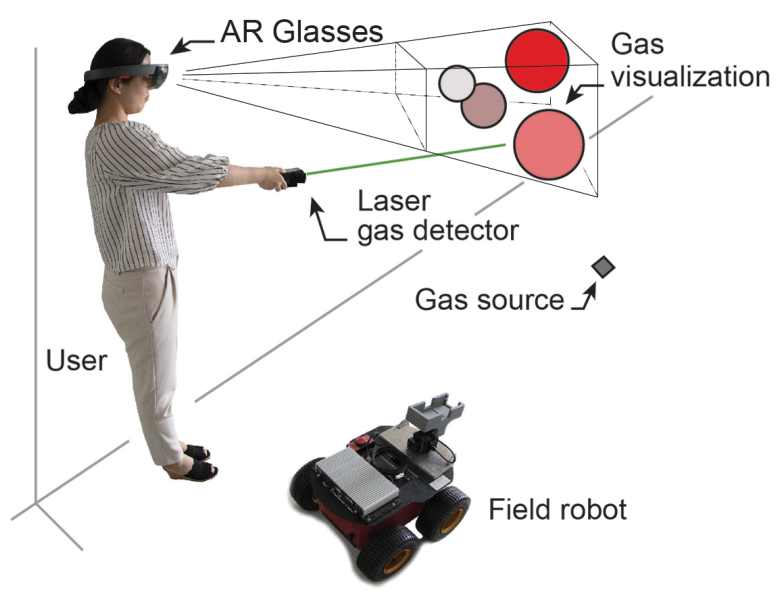

Fig. 1. Concept of our proposed gas visualization model: a user wears the AR glasses while using a gas detector. A detected gas information (e.g., gas concentration and location) are converted into graphics visualization displayed through the AR glasses.

The model measures the gas based on the location where a user points the LMm. Then, the gas distribution is calculated and mapped using visualization parameters before being displayed on the AR-HMD. In the case where the gas location cannot be directly reached by a user, the LMm is mounted on a field robot, and the robot sends information about the gas distribution to the user's AR-HMD.

\section{B. Gas Measurement and Mapping Visualization}

The parameter measured by the LMm is the gas column density, which is the sum of gas concentration per unit area integrated along the laser path. However, the gas concentration on each point is difficult to obtain directly from the LMm. Therefore, we divided the gas field detection area into 3D grids and mapped the gas density of each cell into grids before displaying the gas distribution of the entire field.

To visualize the gas distribution, we considered several characteristics of the gas, such as type, concentration, grid resolution, variance, and position. The type refers to the type of gas detected, i.e., the methane gas; the concentration refers to the volume of the gas or the density of the gas; the variance of the gas is the ability of the gas to spread based on the 


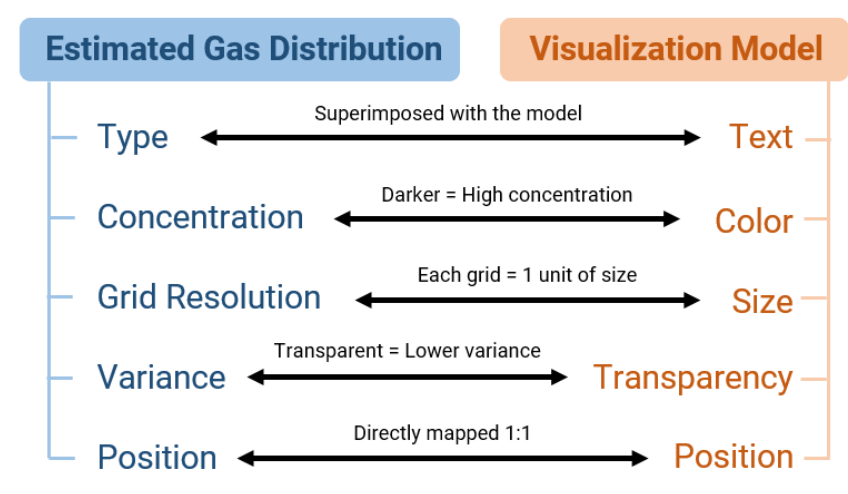

Fig. 2. The relationship between estimated gas distribution and the visualization model.

temperature and wind in the field; and the position of the gas refers to the location where the gas is approximately located.

Using three visualization techniques-bar chart, sphere, and cloud-which represented different data visualization levels (i.e., from abstract to realistic), we mapped the gas distribution with the visualization parameters shown in Fig. 2.

1) Text: Numerous types of gas are displayed as text information next to visualization graphics. Thus, it serves as a direct instruction to a user about the toxicity and harmful effects of the gas.

2) Color: We used the HSL (hue, saturation, lightness), which is a representation of the RGB color model to display the gas concentration. For example, a high gas concentration is displayed with high color intensity (dark color) and vice versa. We mapped the maximum concentration gas to the $L:-100$ (darkness) and vice versa. As a result, measured gas with high concentration is visualized as a dark color, and one with low concentration is visualized as a light color, respectively.

3) Size: We mapped the grid resolution as the size of each visualization technique, as shown in Fig. 3. The larger the gas resolution, the bigger the size of the visualization representation, i.e., the higher the height of the bar chart, the bigger the diameter of the sphere, and the larger the area of the cloud.

The height of the bar chart $V_{\text {height }}$ is calculated based on the gas density $G_{d e n}$ and the maximum gas density $G_{\max }$ in the measurement area, and the maximum height $V_{\max }$ from which a user can observe the visualization of the gas distribution. We formulated a relationship between estimated gas and the visualization techniques as $V_{\text {height }}=\frac{G_{\text {den }}}{G_{\max }} V_{\max }$.

4) Transparency: The transparency level of the visualization is referred to as the variance of the gas, which is affected by atmospheric temperature and wind. The higher the atmospheric temperature, the more spread and opaque the gas visualization and vice versa.

5) Position: The approximate location of the detected gas is mapped to a spatial location of the AR-HMD using a transformation matrix that converts physical locations into spatial locations.
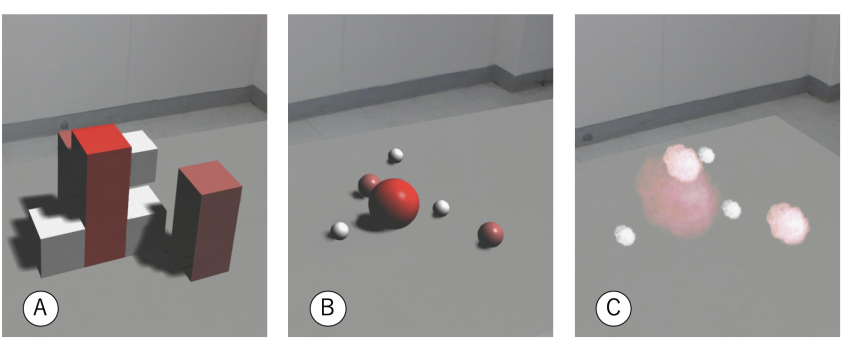

Fig. 3. Our visualization techniques observed from AR glasses; (a) bar chart, (b) sphere, and (c) cloud.

\section{Implementation}

We implemented our visualization techniques and validated their visibility in indoor scene. Since some of the landfill sites are covered with green grass, we used the combination $\mathrm{H}: 0$, S:100, and L:100 to represent the basic colors of the gas visualization, increasing the visibility of the gas.

\section{CONCLUSION AND FUtURE WORK}

In this work, we provided users with all aspects of realtime information using AR technology. The indoor experiments showed different performances of each of the three visualization techniques. We found that when a user stands on the experiment field and observing the gas distribution visualized through the AR-HMD, the cloud visualization technique is the most appropriate technique to use because it could naturally provide detailed information about the gas volume. Conversely, for specific observations, such as the gas maintenance status, the bar chart technique would be the most appropriate technique for archiving and data analysis. In future work, we will experiment and verify our hypothesis in both indoor and outdoor landfill scenarios.

\section{ACKNOWLEDGEMENTS}

This work was supported in part by JSPS KAKENHI Grant Number JP19H02103.

\section{REFERENCES}

[1] M. Inagaki, H. Matsukura, D. Iwai, and K. Sato, "Application of compressed sensing to measurement of methane gas distribution," in Proceedings of the SICE Annual Conference, 2019, pp. 1553-1556.

[2] W. Willett, Y. Jansen, and P. Dragicevic, "Embedded data representations," IEEE Trans. Vis. Comput. Graphics, vol. 23, no. 1, pp. 461-470, 2017.

[3] G. Schall, E. Mendez, E. Kruijff, E. Veas, S. Junghanns, B. Reitinger, and D. Schmalstieg, "Handheld augmented reality for underground infrastructure visualization," Pers. Ubiquitous Comput., vol. 13, no. 4, pp. 281-291, May 2009.

[4] C. Blut and J. Blankenbach, "Three-dimensional citygml building models in mobile augmented reality: a smartphone-based pose tracking system," Int. J. Digit. Earth, to be published.

[5] S. White and S. Feiner, "Sitelens: Situated visualization techniques for urban site visits," in Proceedings of the SIGCHI Conference on Human Factors in Computing Systems, ser. CHI '09. New York, NY, USA: Association for Computing Machinery, 2009, pp. 1117-1120. 